\title{
Research and Development of University Physical Education Cloud Platform Management System Based on Big Data Analysis
}

\author{
Bin Zhang ${ }^{1}$, Bin Fang ${ }^{1}$, Jianwei Yin ${ }^{2}$ and Xiangyang $\mathrm{Yu}^{2^{\star}}$ \\ ${ }^{1}$ Department of General Courses, Jiujiang Vocational and Technical College, Jiujiang 332007, \\ Jiangxi, China \\ ${ }^{2}$ School of Physics, Sun Yat-sen University, Guangzhou 510275, China \\ cesyxy@mail.sysu.edu.cn
}

\begin{abstract}
Keywords: Internet, Cloud platform, Data management System, Big data analysis, University physical education

Abstract. Traditional university physical education (P.E.) will face new challenges and get new development opportunities in the context of the Internet and big data. In this way, it is necessary to establish practical and effective countermeasures. Therefore, we develop a cloud platform management system for university P.E. based on big data analysis model and blockchain technology. It plays a positive role in improving the current state of university P.E. and improving the quality of teaching effects. The management system integrates traditional education management, sports health data analysis, and big data analysis, and attempts to apply the emerging blockchain technology for the first time, so that the data has better security, reliability and effective data reuse.
\end{abstract}

\section{Introduction}

Health is the eternal pursuit of people and the foundation for the full development of mankind. Physical education (P.E.) is a link closely related to people's health and physical fitness. University P.E. is an important part of university education. However, under the current situation of comprehensive implementation of quality education, university P.E. has problems such as unclear teaching objectives, unreasonable teaching content organization, inadequate teaching methods, insufficient scientific assessment methods, and obsolete P.E. equipment. In the context of the rapid development of the Internet, Internet of Things, VR technology, wearable devices and the maturity of these technologies, university P.E. will inevitably present diversified situations, and the corresponding information generated will be more complicated and diversification. The presentation form of information will also be diversified, such as the basic action essentials of a gymnastic action, which can form a multimedia display using text, voice, graphics, still photos, video, or a combination of several forms. In this way, the modern P.E. must be spread from a single message, such as the teacher's classroom teaching, to multiple channels of information dissemination. It will become the main way of college P.E.. In traditional P.E., resource sharing is not realized. If a public university P.E. management platform can be established, hardware, software, data, and the like can be shared, as well as data processing, analysis, and reuse. Therefore, under the background of the Internet, Internet of Things, big data and artificial intelligence, the combination of education and the Internet is the trend of the times. "Internetization" will become a new way for education to transform and change under the new situation. For the education industry, how to apply new technologies and new methods to suit the new situation is a particularly important issue. Under the background of the Internet and big data, university P.E. faces new challenges and opportunities, and it is necessary to establish corresponding countermeasures in the context of new technologies. It will play a significant role in improving the current state of college sports and improving the quality of teaching effects. To this end, we have developed a cloud platform management system for university P.E. based on big data analysis model and blockchain technology. The core idea of this system design is to build a public and open university P.E. management system based on cloud computing, and realize platform sharing, resource sharing and data processing and analysis on the network. Build a high-performance distributed server in the cloud computing center, then develop the corresponding big data processing data model on the 
server, and apply the blockchain technology to process the data to make the data have the blockchain characteristics [1]. This allows anyone, anywhere, and at any time to access the system through a web page for management, teachers, and students [2,3].

\section{Design of Information Management and Data Processing System for University P.E. Cloud Platform}

\section{Demand Analysis}

With the development and changes of the society, people's ideas and needs for education have undergone great changes, the concept and technical means of higher education also need to be developed in time. In university education, P.E. are more related to other courses. In addition to teaching the necessary theoretical knowledge and basic skills, in the curriculum education, effective combination with the students' physical, physical and health management, and further processing the relevant data, such as using the blockchain technology, can give the data new traits and a new role. Such data can be used for specific purposes, such as health, health care, insurance and other projects, to effectively protect the rights of schools and students. This is a comprehensive system engineering. With the development of new technologies, it is possible to establish such a system. There are three groups that need such a system. One is the school, the second is the student, and the third is the data application.

A well-designed cloud platform information management and data processing system must fully guarantee the system functions and consider the factors of the system's capacity, security, and expansion. The platform can obtain a sufficient capacity in the cloud to meet the needs of the system. The network can connect different types of hardware in a scientific and rational allocation of resources and increase the capacity of the system. In order to respond quickly to file data processing requests from different clients, especially when data requests are sent simultaneously from different clients, the system needs corresponding backup data to enhance the security and stability of the system. At the same time, the user's experience should not be affected. The system should have a strong scalability. When the system cannot meet its own needs because of the accumulation of students every year, it does not need to spend more financial and material resources and manpower to rebuild the system. It only needs to develop a simple expansion on the original basis. Therefore, the platform should be fully evaluated in terms of compatibility between system hardware and software design.

When analyzing different user needs, we need to analyze the functional requirements of administrators and different users. Whether for administrators or ordinary users, it is necessary to achieve data file compression, transmission, query, modification and other operational functions. For the administrators, permissions are larger than the users, need to achieve more functions than the users, such as the implementation of the audit of the users and querying the data files of the users.

\section{System Design Goal}

A complete data information management system, while fully ensuring the realization of system functions, must also consider the factors of the system's own capacity, security, and expansion. A large-capacity system is in need that can scientifically allocate different types of hardware resources through network connections and increase the capacity of the system. For file data processing requests from different clients, it is necessary to be able to respond quickly, especially when different data requests are sent from different clients. The entire system should be able to be processed in parallel. In the event of virus intrusion or data corruption caused by some hardware resources, the system needs corresponding backup data, and the original data backup is used to reinforce the security and stability of the system, and at the same time, it will not bring a poor response experience to the user. The system itself must have strong scalability. As the size of all kinds of students is so large that the system cannot meet its own needs, it does not need to spend more financial and material resources to re-establish the update system, only need to expand on the original basis. Therefore, we must fully consider the compatibility of system hardware and software [4]. 


\section{System Architecture}

Fig. 1 shows the overall architecture of the university P.E. cloud platform management system. It is a cloud-centric, public, open cloud platform management system. It is a machine learning-based web application developed in Java. The algorithm is based on the open source Python machine learning library Scikit-Learn. The database uses MySQL. It is mainly used for teaching management, training models, data sets and test results, as well as statistical data such as user download information and user data analysis.

\section{Main Function of Each Module}

Users can access the network by wired or wireless means, and the functions of the system can be used, which saves the cost of the terminal equipment. The system consists of four modules: teaching data management, sports health data management, data analysis and processing, and system management. The main functions and functions of each module are introduced below [5].

\section{Teaching Data Management}

This module combines the university's general educational management functions and further links with the functions of the smart campus according to the characteristics of the university P.E.. There are mainly the following aspects of the function.

(1) Educational Administration System: It connects with the school's academic department to complete daily teaching and related functions of students, such as course selection, attendance, and performance management. And it generates relevant data, which is included in the big data of the system.

(2) Video teaching system: It connects the public and school video teaching resource library. At the same time, it provides a video teaching interface for humanized teaching.

(3) Campus Activity Management System: An important content of university P.E. is not only in classroom teaching, but also in various activities of the campus, such as sports games, club activities, and literary activities. It is also the main body of various international, national, and provincial-level competitions. Therefore, a campus activity management system is established to effectively manage the student's campus activities. Through the effective link with the data processing module and using the blockchain, the records of student participation in the activity have credibility and become part of evaluating the extracurricular achievements and abilities.

\section{Sports Health Data Management}

This module implements two main functions. One is related to public health platforms, student hospitals, and emergency systems to establish a student's physical foundation database. The other is monitoring sports data. Data sources can be divided into two categories. One is the physical index data in the classroom, which is extremely important for effectively designing the amount of classroom exercise. The other is that with the development of wearable sports equipment, the data of normal sports is uploaded to the system and becomes part of the system data. This part is divided into the following three submodules.

(1) Public health platform: It is associated with public health networks, school hospitals, etc.

(2) Basic health data: It manages and analyzes student basic physical data such as experience and visits.

(3) Sports monitoring data: The monitoring of classroom physical indicator data and the recording of students' own exercise data.

\section{Data Analysis and Processing}

This module is mainly used for data analysis and processing. It mainly includes the following submodules [6].

(1) Statistical analysis: This part is the processing of big data, including statistical analysis of big data in the recording, storage and management.

(2) Access statistics: The module contains statistics and analysis of the user's information about downloading and uploading various types of data. 
(3) Blockchain technology processing: All recorded data is processed by blockchain technology, so that all of the system has blockchain characteristics.

\section{System Management}

This is an auxiliary module of the system. The main functions are as follows.

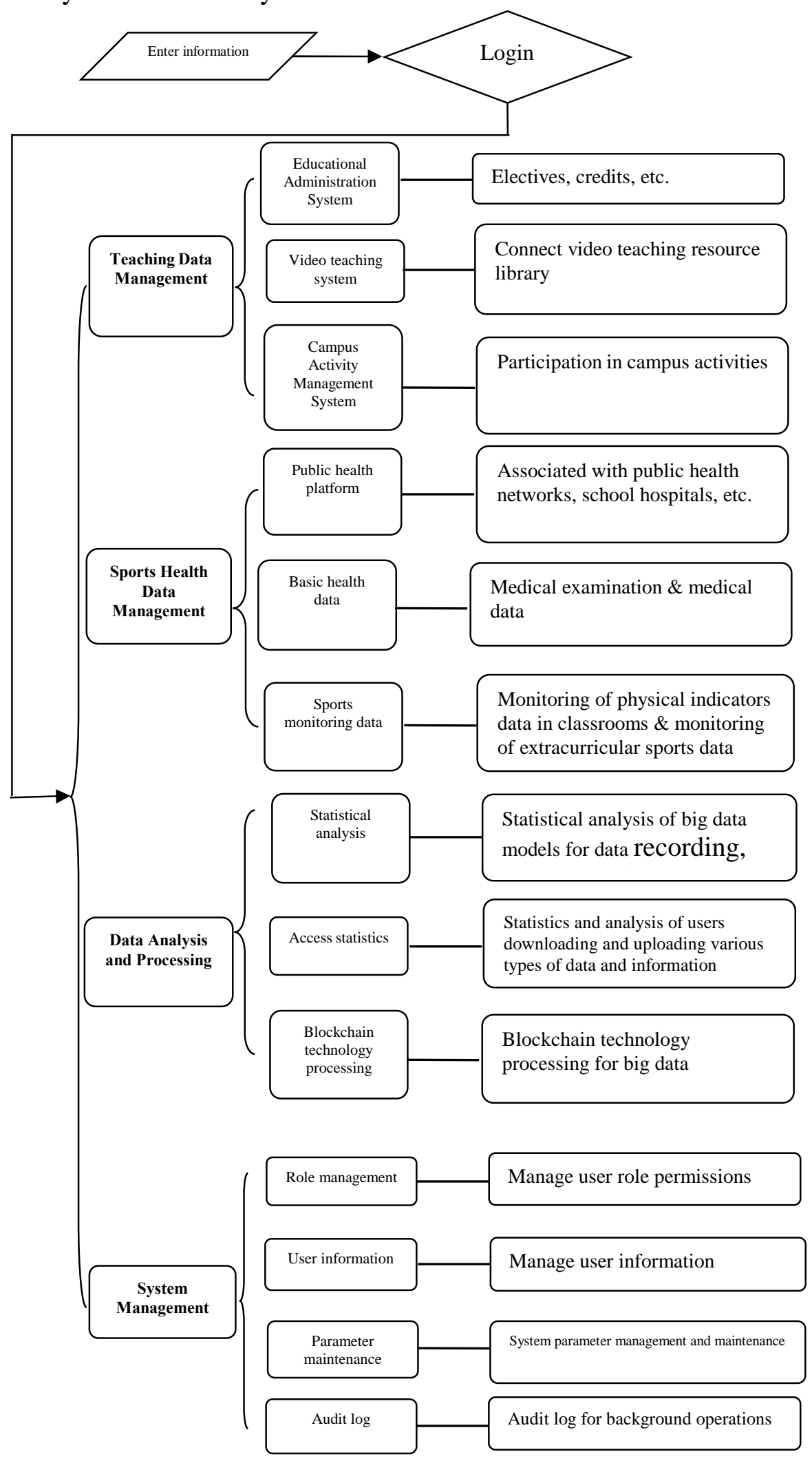

Fig.1 System architecture and functionality

(1) Role management: Role permissions. The main user roles are students, teachers, visitors, system administrators, system maintainers. 
Manage the user's role management in the background. It is mainly the role and authority management of the background management personnel who can control them flexibly and dynamically.

(2) User information: This module is mainly used for information query and blacklist management of registered users, mainly including the functions of querying and exporting information, and adding and removing blacklists.

(3) Parameter maintenance: Parameter maintenance is mainly the setting and modification of some system parameters.

(4) Audit log: The audit log of the background operation, including the addition, deletion, and change of the administrator operation log.

\section{Conclusion}

The era of big data has arrived. Although big data has become a hot topic in many research fields including P.E., it is rare to find the P.E. management system based on big data technology and blockchain technology. The research is still in a very preliminary stage, and there are still many basic problems to be solved and studied. The development and implementation of various P.E. programs in the era of big data will be based more on data, analysis and facts than on experience and intuition. Therefore, under the current conditions, although the application of big data in P.E. has certain difficulties, the establishment of information systems including teaching, sports and health is urgently solved. Teaching and student historical data is either discarded, incomplete or difficult to obtain publicly. The lack of data and the difficulty of obtaining data make it difficult to obtain meaningful information from these data. There is still a long way to go to solve all kinds of big data problems in P.E.. Therefore, we are trying to develop a university P.E. cloud platform management system based on big data analysis developed on a cloud server. It is expected to provide a certain reference and practice platform for P.E. teachers and university students to adapt to the development of modern science and technology, so that the quality and effect of university P.E. can be upgraded to a new level to expand the function of P.E.. Through the actual use and testing, the application process of the system in teaching is gradually improved and matured.

\section{References}

[1] Shen, Zhonghua, et al., A Probe into the Education Big Data and Educational Evaluation from the Perspective of New Technology: Also on the Influence of Block Chain Technology on the Evaluation of Online Education. Journal of Distance Education, 2017.

[2] George, Gerard, Martine R. Haas, and Alex Pentland, Big data and management, 2014, p. 321-326.

[3] Liu P T S, in: Medical record system using blockchain, big data and tokenization. International Conference on Information and Communications Security. Springer, Cham, 2016, p. 254-261

[4] McLeod R, Schell G P, Management information systems. USA: Pearson/Prentice Hall, 2007.

[5] Hirsch B, Ng J W P, Education beyond the cloud: anytime-anywhere learning in a smart campus environment. Internet Technology and Secured Transactions (ICITST), 2011 International Conference for. IEEE, 2011, p. 718-723.

[6] Chen C L P, Zhang C Y, Data-intensive applications, challenges, techniques and technologies: A survey on Big Data. Information Sciences, 2014, 275: p. 314-347. 